\title{
Terminal regions of chromosome arms 6AL and 6BL carry QTL affecting seminal root angle in wheat (Triticum aestivum L.)
}

\author{
Masahiko Mori $^{1,2}$, Atsushi Oyanagi ${ }^{1,3}$, Emdadul Haque ${ }^{1,3}$, Kentaro Kawaguchi ${ }^{1,4}$, \\ Hideho Miura ${ }^{2}$ and Fumitaka Abe ${ }^{1}$ \\ ${ }^{1}$ Institute of Crop Science, NARO, Tsukuba, Ibaraki 305-8518, Japan \\ ${ }^{2}$ Obihiro University of Agriculture and Veterinary Medicine, Obihiro, Hokkaido 080-8555, Japan \\ ${ }^{3}$ Present address: Kyushu Okinawa Agricultural Research Center, NARO, Miyakonojo, Miyazaki 885-0091, Japan \\ ${ }^{4}$ Present address: Genetic Resources Center, NARO, Tsukuba, Ibaraki 305-8602, Japan
}

Corresponding author: M. Mori, E-mail: mmmori@obihiro.ac.jp, phone: +81-155-49-5486, Fax:+81-155-49-5593

Received on June 25, 2020; Accepted on October 16, 2020

\begin{abstract}
The identification of genetic factors affecting deep root systems is needed to improve adaptability to drought stress. In wheat (Triticum aestivum $\mathrm{L}$.), seminal root angle (SRA), which can be easily measured at the seedling stage, is a useful proxy for understanding the mature root system architecture. In the present study, we attempted to find a genetic variation in the vertical distribution of seminal roots and to determine chromosomal positions involved in a variation of SRA using ditelosomic (Dt) lines and deletion lines of wheat cultivar Chinese Spring (CS). SRA of CS and 31 Dt lines were estimated in a pot experiment using the basket method. When SRA was measured separately for the primary, first pair, and second pair of seminal roots, it was found that the genetic variation was the largest in the first pair of seminal roots followed by the second pair of seminal roots. In contrast, there were no genetic differences in the SRA of the primary root between the CS and CS Dt lines. Compared to CS, which had a small SRA, Dt5BL, Dt6AS, Dt6BS, and Dt6DS displayed significantly larger SRA, indicating that the deficient chromosome arms 5BS, 6AL, 6BL, and 6DL appeared to carry quantitative trait loci (QTLs) affecting SRA. Furthermore, the terminal regions of chromosome arms $6 \mathrm{AL}$ and $6 \mathrm{BL}$ were found to carry QTLs responsible for a small SRA. Using the QTLs information obtained in this study, novel genes may be isolated from wheat.
\end{abstract}

Keywords: quantitative trait loci, root system architecture, seminal root angle, Triticum aestivum $\mathrm{L}$.
Abbreviations: QTLs, quantitative trait loci; SRA, seminal root angle; Dt, ditelosomic lines

\section{Introduction}

Wheat (Triticum aestivum L.) is one of the most important food crops in human diet. Wheat is mainly grown under rain-fed agricultural regions of the world. Under rain-fed conditions, wheat growth is often inhibited throughout their growing season, that is, their vegetative, reproductive, and grain filling periods, owing to drought stress. Drought stress, which is one of the most important factors influencing wheat production in the semi-arid regions of the world, has been reported to cause a significant decline in wheat yield (Passioura 2006, Bassi and Sanchez-Garcia 2017). Therefore, enhancing the adaptation of wheat to drought stress is a major objective in the world.

Root system architecture plays an important role for crops grown in various soil moisture environments. In general, compared to crops with shallow root systems, those with deep root systems maintain higher yields under water-limiting environments owing to better access to water in deep soil layers (Manschadi et al. 2008, Haque et al. 2016). This implies that varieties with deeper root systems might be adapted to drought stress compared to varieties with shallower root growth. Therefore, the identification of genetic factors affecting deep root systems is needed to improve adaptability to drought stress.

The root system of wheat consists of two main

Mori M, Oyanagi A, Haque E, Kawaguchi K, Miura H and Abe F 2020 Terminal regions of chromosome arms 6AL and 6BL carry QTL affecting seminal root angle in wheat (Triticum aestivum L.). Plant Root 14:23-31. doi:10.3117/plantroot.14.23 
roots, which are the seminal roots and the nodal roots (Chochois et al. 2015). A wheat seedling has five seminal roots that are formed from one primary seminal root, two first pairs of seminal roots, and two second pairs of seminal roots. After germination, these roots emerge in an order with primary seminal root, first pair of seminal roots, and second pair of seminal roots. Nodal roots appeared at the tillering stage. Seminal roots reach deep soil layers earlier than the nodal roots (Manschadi et al. 2013). Large seminal root angle (SRA) promotes deeper root growth and often improves access to water. In durum and bread wheat, SRA, which can be easily measured at the seedling stage, is representative of the mature root system architecture (Fang et al. 2017, Hassouni et al. 2018). A close positive correlation was reported between root depth index in the field and SRA in a pot experiment (Oyanagi 1994). Manschadi et al. (2008) demonstrated that SRA varied between Australian wheat varieties and correlates strongly with differences in mature root system distribution. These results suggested that SRA is a useful indicator of the characteristics of vertical distribution of roots (Oyanagi et al. 1993).
Quantitative trait locus (QTL) analysis for SRA and related traits has been conducted in durum and bread wheat. In durum wheat, five QTLs for SRA were mapped on the chromosome arms 3AS, 4AL, 5BS, 5BL, and 6AS (Sanguineti et al. 2007). Maccaferri et al. (2016) detected nine main QTL clusters on chromosomes $2 \mathrm{~A}$ (two regions), 2B, 4B, $6 \mathrm{~A}$ (two regions), 7A (two regions), and 7B using two recombinant inbred line populations and the association panel in durum wheat. Furthermore, the major QTL ( $q S R A-6 A)$ detected in the distal end of chromosome arm 6AL was co-located with previously reported durum QTL for root-growth angle (Alahmad et al. 2019). In bread wheat, four QTLs for SRA were identified on chromosomes 2A, 3D, 6A, and 6B (Christopher et al. 2013). Two QTLs for deep root ratio were reported on chromosomes 1B and 5D by Hamada et al. (2012), although they did not identify QTL for SRA per se.

In the present study, we attempted to 1) find genetic variation in vertical distributions of seminal roots and to 2) determine chromosomal positions of possible QTL involved in a variation of SRA using ditelosomic (Dt) lines and deletion lines with

Table 1. Ditelosomic lines used for measurement of seminal root angle (SRA)

\begin{tabular}{|c|c|c|c|}
\hline \multicolumn{2}{|c|}{ Deletion of long arm chromosome } & \multicolumn{2}{|c|}{ Deletion of short arm chromosome } \\
\hline NBRP ID & Line name & NBRP ID & Line name \\
\hline \multirow[t]{2}{*}{ LPGKU2001 } & CS Ditelosomic 1AS (Dt1AS) & LPGKU2002 & CS Ditelosomic 1AL (Dt1AL) \\
\hline & & LPGKU2004 & CS Ditelosomic 1BL (Dt1BL) \\
\hline LPGKU2005 & CS Ditelosomic 1DS (Dt1DS) & LPGKU2006 & CS Ditelosomic 1DL (Dt1DL) \\
\hline \multirow[t]{3}{*}{ LPGKU2007 } & CS Ditelosomic 2AS (Dt2AS) & & \\
\hline & & LPGKU2010 & CS Ditelosomic 2BL (Dt2BL) \\
\hline & & LPGKU2012 & CS Ditelosomic 2DL (Dt2DL) \\
\hline LPGKU2013 & CS Ditelosomic 3AS (Dt3AS) & LPGKU2014 & CS Ditelosomic 3AL (Dt3AL) \\
\hline \multirow[t]{3}{*}{ LPGKU2015 } & CS Ditelosomic 3BS (Dt3BS) & LPGKU2016 & CS Ditelosomic 3BL (Dt3BL) \\
\hline & & LPGKU2018 & CS Ditelosomic 3DL (Dt3DL) \\
\hline & & LPGKU2020 & CS Ditelosomic 4AL (Dt4AL) \\
\hline LPGKU2021 & CS Ditelosomic 4BS (Dt4BS) & & \\
\hline \multirow[t]{4}{*}{ LPGKU2023 } & CS Ditelosomic 4DS (Dt4DS) & LPGKU2024 & CS Ditelosomic 4DL (Dt4DL) \\
\hline & & LPGKU2026 & CS Ditelosomic 5AL (Dt5AL) \\
\hline & & LPGKU2028 & CS Ditelosomic 5BL (Dt5BL) \\
\hline & & LPGKU2030 & CS Ditelosomic 5DL (Dt5DL) \\
\hline LPGKU2031 & CS Ditelosomic 6AS (Dt6AS) & LPGKU2032 & CS Ditelosomic 6AL (Dt6AL) \\
\hline LPGKU2033 & CS Ditelosomic 6BS (Dt6BS) & & \\
\hline LPGKU2035 & CS Ditelosomic 6DS (Dt6DS) & LPGKU2036 & CS Ditelosomic 6DL (Dt6DL) \\
\hline LPGKU2037 & CS Ditelosomic 7AS (Dt7AS) & LPGKU2038 & CS Ditelosomic 7AL (Dt7AL) \\
\hline LPGKU2039 & CS Ditelosomic 7BS (Dt7BS) & LPGKU2040 & CS Ditelosomic 7BL (Dt7BL) \\
\hline LPGKU2041 & CS Ditelosomic 7DS (Dt7DS) & LPGKU2042 & CS Ditelosomic 7DL (Dt7DL) \\
\hline LPGKU2269 & Chinese Spring $(\mathrm{CS})$ & & \\
\hline
\end{tabular}


uniform genetic background of wheat cultivar Chinese Spring (CS).

\section{Materials and Methods}

\section{Plant materials}

In the first experiment, CS and CS Dt lines were used. CS was the standard variety of the genetic analysis. The CS Dt lines developed by Sears (1954) have 20 pairs of normal chromosomes and one pair of chromosomes with either the short arm or the long arm deleted. Of possible CS Dt lines, 11 lines were not available owing to low fertility or poor growth. Thus, CS and 31 CS Dt lines were used in the present experiment (Table 1).

To determine the chromosomal positions of possible QTLs for SRA detected in the CS Dt lines experiment, we used further three deletion lines of chromosome 6AL (6AL-08, 6AL-01, and 6AL-06) and three of 6BL (6BL-01, 6BL-03, and 6BL-12). These CS deletion lines had different fraction lengths (FL) (Table 2) that showed relative length of the long arm (Endo and Gill 1996).

\section{Plant growth condition and measurement of average SRA}

The SRA of CS and 31 CS Dt lines were estimated in a pot experiment using the basket method (Oyanagi 1994) with slight modifications. We used a plastic meshwork basket with an open top diameter of $160 \mathrm{~mm}$ and a flat bottom diameter of $85 \mathrm{~mm}$, in which the plants were cultured within a $55 \mathrm{~mm}$ depth (BB-15B, Yazaki Kako Corporation, Shizuoka, Japan). The plastic meshwork basket, filled with $600 \mathrm{~mL}$ of granular soil (Shibanome soil, Kanuma Kousan Co., Tochigi, Japan; particles 1-3 $\mathrm{mm}$ in size, no fertilizer, $\mathrm{pH} 5.6-6.0$ ), was placed in a 1/5000 a Wagner's pot (Fujiwara Scientific Co. Ltd, Tokyo, Japan). Seeds were sterilized in a $1 \%$ sodium hypochlorite solution for $10 \mathrm{~min}$ and rinsed with tap water for $15 \mathrm{~min}$. The sterilized seeds were incubated at $20^{\circ} \mathrm{C}$ in the dark until the radicles emerged. Three germinated seeds were planted in the center of each basket and this was replicated four times. The seeds were covered with a $10-\mathrm{mm}$ layer of soil. The pot was placed in a plastic container and supplied with tap water. The water level in the plastic container was maintained at 40-mm depth. The plants were irrigated everyday with tap water to prevent root drying. They were grown in a greenhouse maintained at $23^{\circ} \mathrm{C}$ and with 12 (late March) to 14 hours (late May) of natural daylight.

The SRA in CS and each CS Dt lines were
Table 2. CS deletion lines used for determination of chromosomal positions of possible QTL for SRA

\begin{tabular}{ccc}
\hline NBRP ID & Line name & Fraction length $^{\mathrm{a}}$ \\
\hline LPGKU1244 & CS Deletion 6AL-08 & 0.90 \\
LPGKU1241 & CS Deletion 6AL-01 & 0.69 \\
LPGKU1242 & CS Deletion 6AL-06 & 0.57 \\
LPGKU1247 & CS Deletion 6BL-01 & 0.70 \\
LPGKU1248 & CS Deletion 6BL-03 & 0.36 \\
LPGKU1256 & CS Deletion 6BL-12 & 0.05 \\
\hline
\end{tabular}

${ }^{a}$ The position of break point from the centromere relative to the length of the complete arm.

measured on the $14^{\text {th }}$ day after planting. For individual seminal roots, SRA was determined by measuring the angle between the horizontal and the line connecting the root penetration site and the center of the basket surface using a protractor with $10^{\circ}$ increments. The SRA of CS and each CS Dt were calculated from an average value of 60 angle data: 3 plants $\times 5$ seminal roots $\times 4$ replications. The number of seminal roots of the 12 plants per line was also recorded.

\section{Measurement of SRA in individual roots}

In the previous reports, SRA of bread and durum wheat seedling was suggested to be larger in the primary seminal root than the first pair of seminal roots (Oyanagi 1994, Alahmad et al. 2019). So, we separately measured SRA of the primary seminal root and the first and second pairs of seminal roots in $31 \mathrm{CS}$ Dt lines, CS deletion 6AL, and CS deletion 6BL using the basket method as described above. In this experiment, to avoid root getting entangled, one seed was planted per basket. Fourteen days after planting, SRA of each root was measured using a protractor with $5^{\circ}$ increments and each root was marked to distinguish individual roots. The plant was pulled out from the basket maintained under water to avoid root injury. All roots were separated into the primary, first pair, and second pair of seminal roots. SRA of two roots of the first pair and second pair of seminal roots was averaged. The experiments were carried out with three (CS Dt experiment) and nine (CS deletion experiment) replications.

\section{Statistical analysis}

Dunnett's multiple comparison tests were used for detecting significant differences $(P<0.05)$ in SRA traits between CS and each of the CS Dt lines, and also to test for differences between CS and deletion lines. 


\section{Results}

\section{Variation of SRA and seminal root number}

At first, root system architecture was estimated using the average value of all SRA of the primary, first pair, and second pair of seminal roots. The average SRA of CS was $42.7^{\circ}$ and that of CS Dt lines varied from $40.5^{\circ}$ to $68.8^{\circ}$ in Dt7DL and Dt6AS, respectively (Fig. 1a). Four CS Dt lines, namely Dt5BL, Dt6AS, Dt6BS, and Dt6DS, had significantly larger average SRA than CS (Fig. 1a). Figure 2 shows seminal root phenotypes as observed in the basket method: CS has shallow root architecture compared to Dt6AS. There were no lines that had smaller SRA than CS.

In the present pot experiment, the number of seminal roots per plant ranged from three to five (Fig. 1b). Three lines, namely Dt2BL, Dt6AS, and Dt6BS, could not develop the second pair of seminal roots. Some variations in seminal root number among plants within a line was also observed. Most of these variations were due to missing of the second pairs of seminal roots. There was no evidence for a significant genetic relationship between average SRA and seminal root number because correlation between these two traits was not detected $(r=-0.29)$.

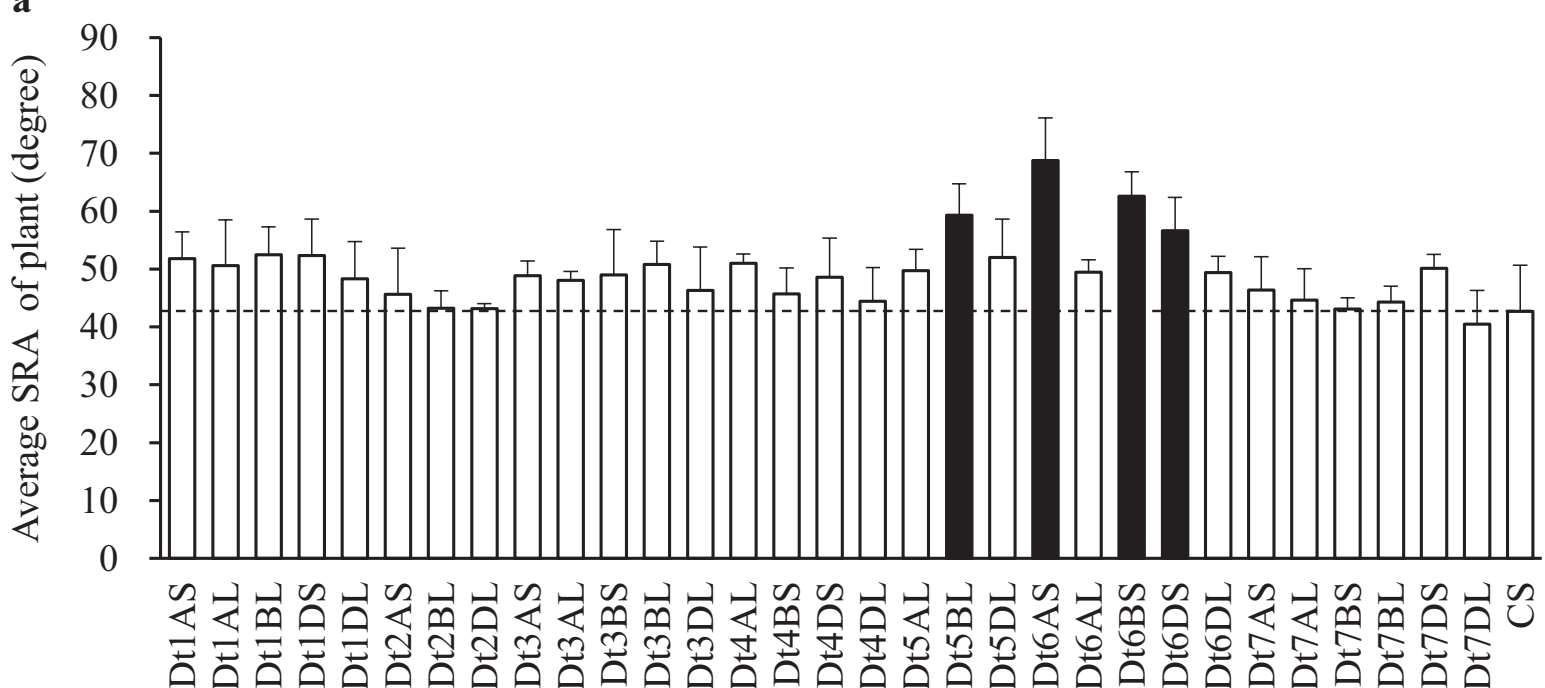

b

Line

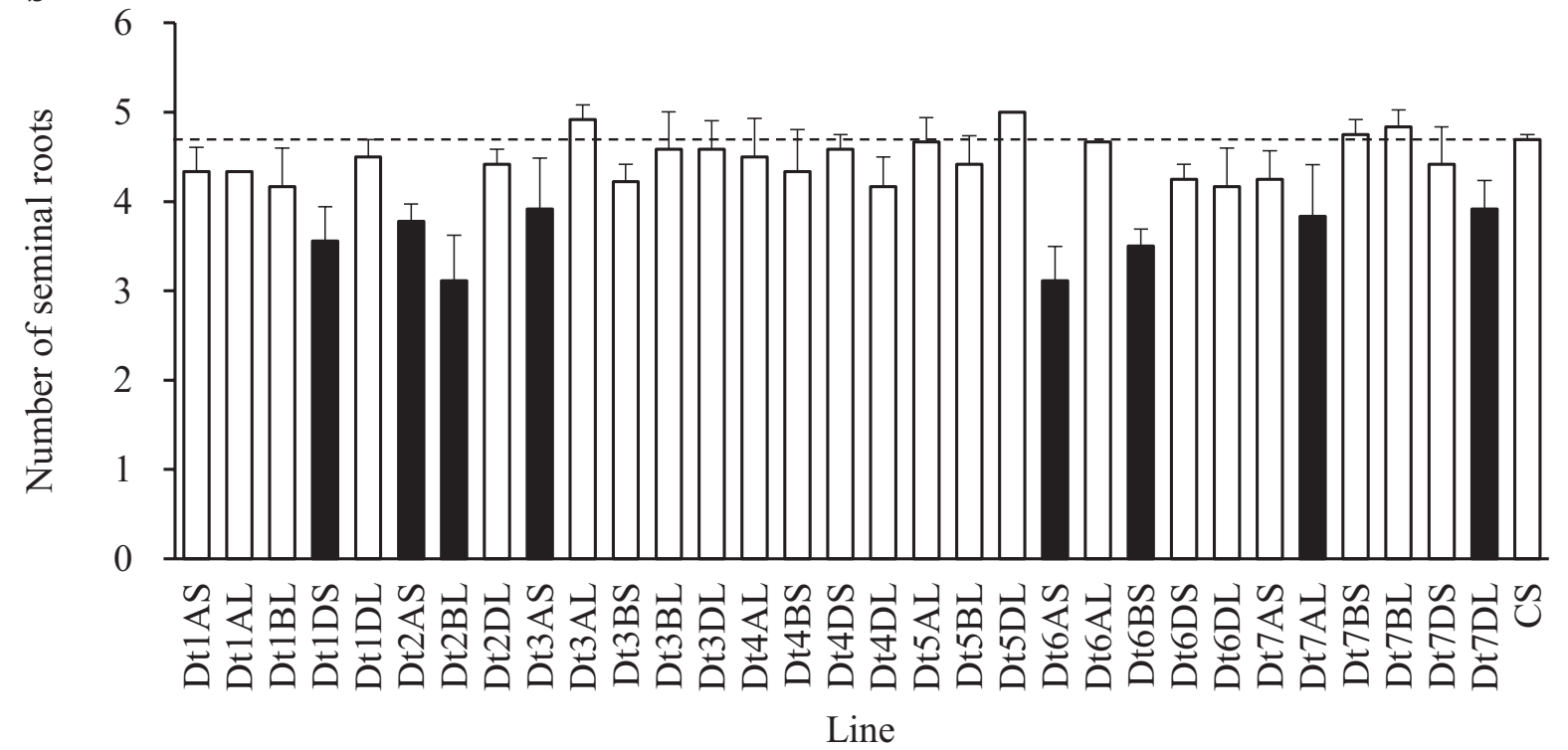

Fig. 1. Seminal root traits for CS and 31 CS Dt lines measured by the basket method. Average SRA of plant (a) and number of seminal roots per plant (b). Dt lines with solid bars indicate significant difference from CS at the 5\% level (Dunnett's test). The error bars represent standard deviation. The dashed line shows the mean value of CS. 


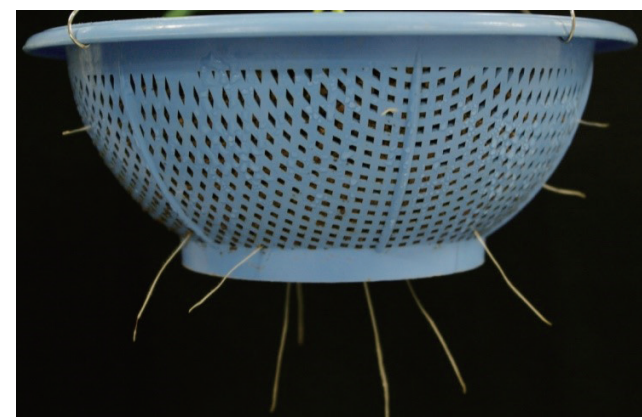

b

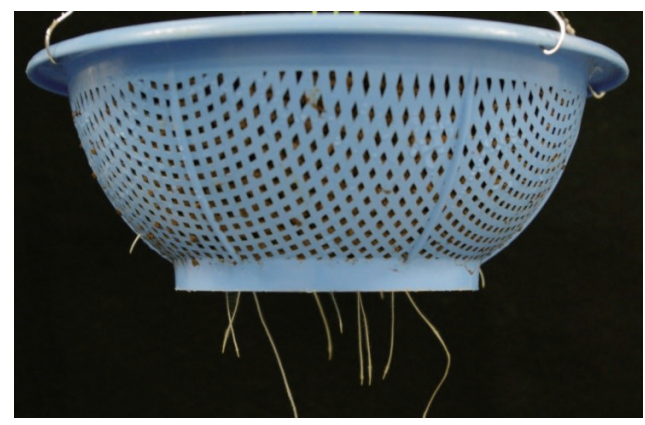

Fig. 2. Seminal root phenotypes of CS (a) and Dt6AS (b) in the basket method.

\section{SRA of individual roots in CS Dt lines}

SRA for individual roots in CS Dt lines were given in Fig. 3. In all lines, SRA for the primary seminal root was the largest $\left(\right.$ mean $\left.=70.1^{\circ}\right)$, followed by that for the first pair $\left(\right.$ mean $\left.=49.2^{\circ}\right)$ and second pair of seminal roots $\left(\right.$ mean $\left.=23.6^{\circ}\right)$. The primary seminal root of CS and CS Dt lines extended more to the vertical direction and the variation in CS Dt lines was small (Fig. 3a).

SRA for the first pair of seminal roots ranged from $30.8^{\circ}$ in Dt4DL to $67.5^{\circ}$ in Dt5AL, while SRA for the second pair of seminal roots ranged from $6.3^{\circ}$ in Dt2DL to $46.7^{\circ}$ in Dt6AS (Fig. $3 b$ ). Compared to CS $\left(43.3^{\circ}\right)$, six CS Dt lines (Dt1AS, Dt5AL, Dt5BL, Dt6AS, Dt6BS, Dt6DS) showed larger SRA of the first pair of seminal roots $(P<$ 0.05 ). There were no lines that had significantly smaller SRA or wider root angle of the first pair of seminal roots compared to CS.

For the second pair of seminal roots, Dt5BL, Dt6AS, and Dt6DS showed statistically larger SRA than did CS $(P<0.05)$ (Fig. 3c).

\section{Relationship among SRA of individual roots}

Correlations among SRA of individual roots were examined (Fig. 4). SRA of the primary root was not correlated with SRA of the first pair of seminal roots and with the second pair of seminal roots. In contrast, there was a significant correlation between SRA of the first pair of seminal roots and SRA of the second pair of seminal roots $(\mathrm{r}=0.45, P<0.05)$.

\section{Estimation of QTLs for SRA of seminal roots}

For the first pair of seminal roots, Dt1AS, Dt5AL, Dt5BL, Dt6AS, Dt6BS, and Dt6DS displayed significantly larger SRA than did CS. This result was repeatable in both trials, except for Dt1AS and Dt5AL (Fig. 1a, Fig. 3b). Therefore, the deficient chromosome arms 5BS, 6AL, 6BL, and 6DL appear to carry QTLs affecting root growth angle especially favoring shallow growth in the first pair of seminal roots. On the contrary, Dt2BL and Dt6BS could not produce the second pair of seminal roots, suggesting a possibility that chromosome arms $2 \mathrm{BS}$ and $6 \mathrm{BL}$ could possess QTL that promote development of the second pair of seminal roots.

Estimation of QTL position on the long arms of chromosomes $6 A$ and $6 B$

To confirm the position of QTLs, which were possibly located on the long arms of chromosomes 6A and 6B, we used three CS6AL and three CS6BL deletion lines. From the above-mentioned result of CS Dt lines, the genetic difference for SRA was more evident in the first pair of seminal roots. Hence, we analyzed this seminal root (Fig. 5). The SRA of 6AL-08, 6AL-01, and 6AL-06 were identical to that of Dt6AS but significantly larger than that of CS. A similar result was observed in the three deletion lines for chromosome arm 6BL where the SRA of $6 \mathrm{BL}-01,6 \mathrm{BL}-03$, and $6 \mathrm{BL}-12$ were around $50^{\circ}$ and identical to that of Dt6BS $\left(53.1^{\circ}\right)$ but significantly greater than that of CS. The effect of chromosome arm 5BS was not further examined owing to the lack of adequate deletion lines.

Consequently, we concluded that the terminal regions of chromosome arms 6AL $(\mathrm{FL}=0.9-1.0)$ and 6BL (FL $=0.70-1.0)$ of CS carried QTLs responsible for a small SRA (Fig. 5).

\section{Discussion}

In the present study, we found a large variation of SRA for the first pair of seminal roots among Dt lines. With this result, we identified QTLs affecting SRA of the first pair of seminal roots in CS on the short arm of chromosome 5B (designated as QSra. $o b u-5 B S$ ) and on the long arms of chromosomes $6 \mathrm{~A}$ (designated as QSra.obu-6AL), 6B (designated as QSra.obu-6BL), and 6D (designated as QSra.obu$6 D L)$. In addition, QTLs associated with a small SRA of CS were detected at the terminal regions of 
a

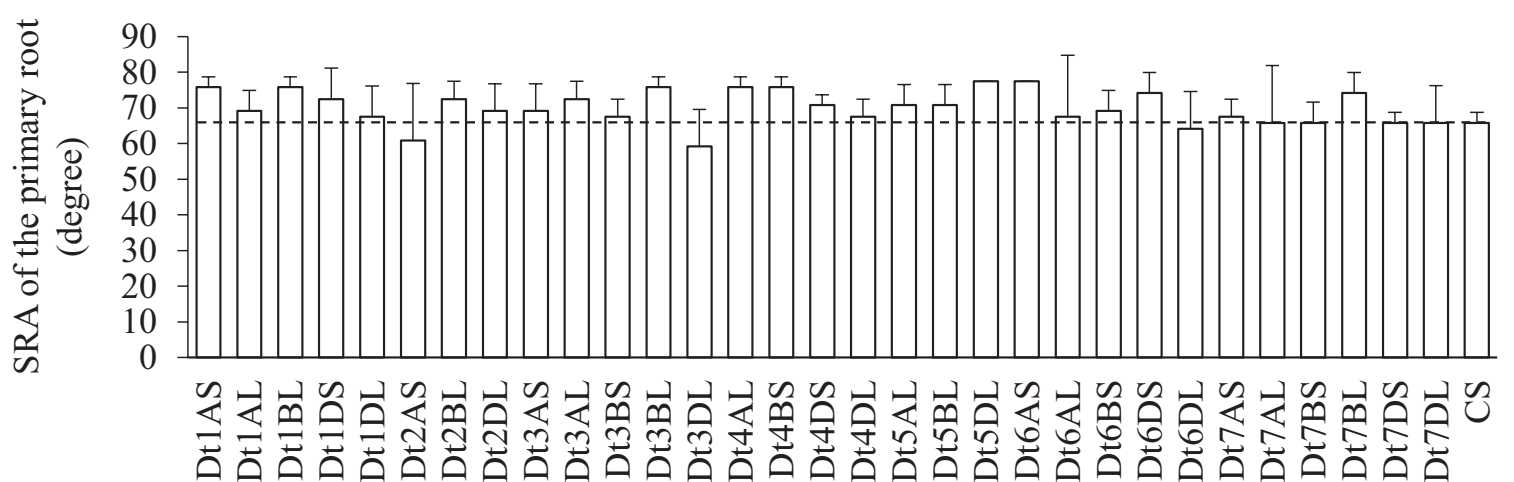

b

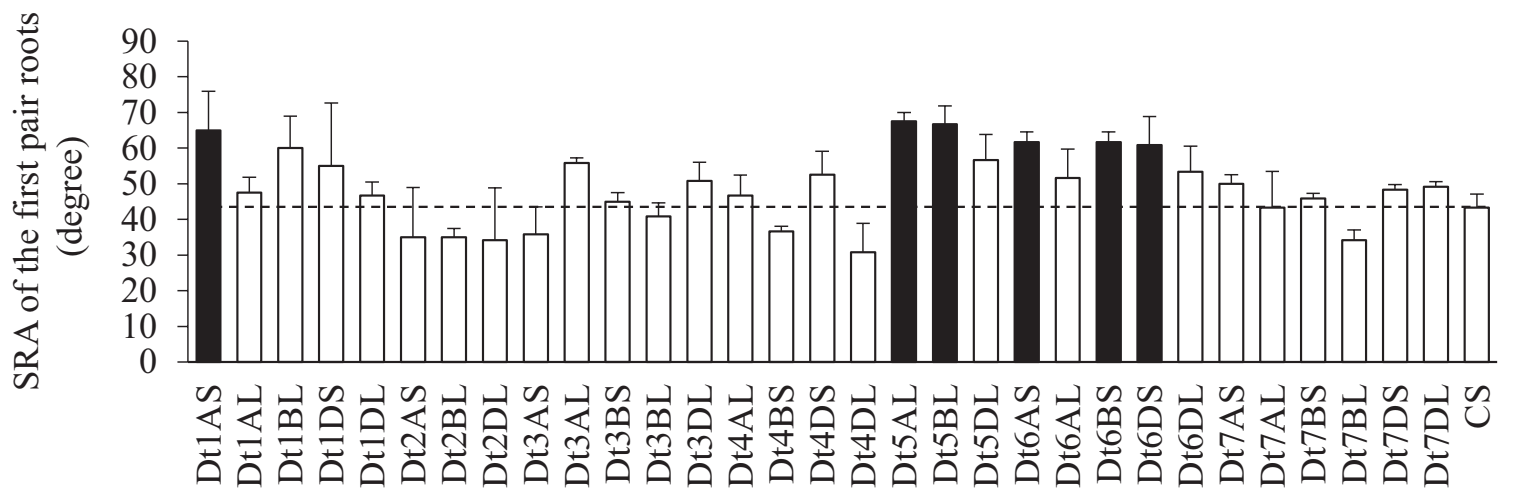

c

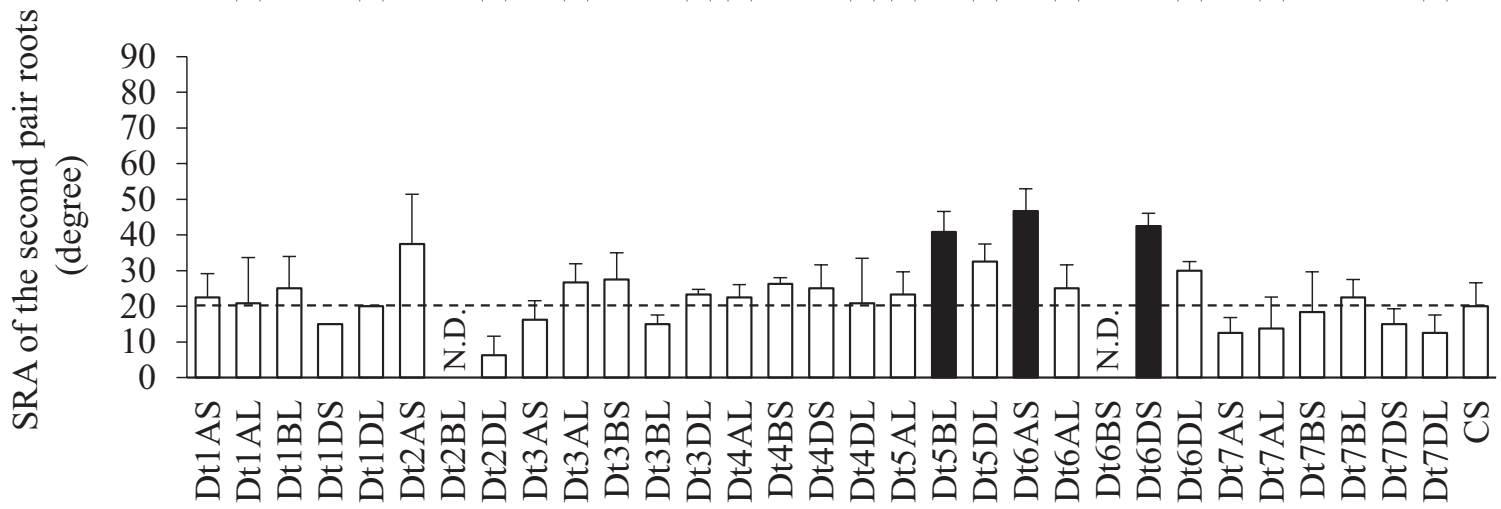

Line

Fig. 3. SRA of the primary seminal root (a) and first (b) and second pairs of seminal roots (c) in CS and 31 CS Dt lines. Dt lines with solid bars indicate significant difference from CS at the $5 \%$ level (Dunnett's test). The error bars represent standard deviation. The dashed line shows the mean value of CS. N.D. means not determined.
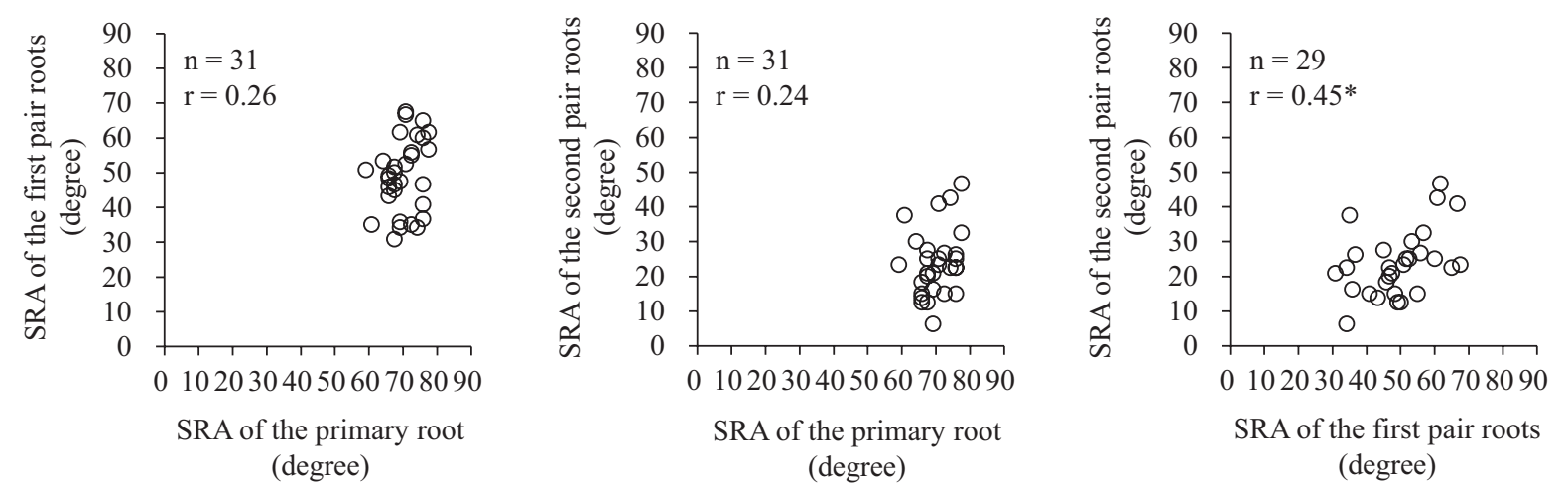

Fig. 4. Relationships among SRA of individual roots in CS Dt lines. * indicates significant correlation at the $5 \%$ level. 
chromosome arms 6AL and 6BL.

It is well known that there are varietal differences in SRA at the seedling stage in bread wheat (Oyanagi et al. 1993, Manschadi et al. 2008, Hamada et al. 2012, Christopher et al. 2013). These variation of SRA were observed for the average of SRA in plants (Oyanagi et al. 1993, Hamada et al. 2012) or SRA in individual roots (Manschadi et al. 2008, Christopher et al. 2013). In this study, it was shown that the variation of SRA in individual roots among CS Dt lines was larger than that of the average SRA in plants (Fig. 1a, Fig. 3). The primary seminal root of CS and CS Dt lines extended more to the vertical direction but the variation in $\mathrm{CS} \mathrm{Dt}$ lines was small, ranging from $59.2^{\circ}$ to $75.8^{\circ}$ (Fig. $3 a)$. On the contrary, the variation among the lines in SRA for the first pair of seminal roots was much larger than that of the primary seminal root, ranging from $30.8^{\circ}$ to $67.5^{\circ}$ (Fig. 3b). A similar result was reported by Manschadi et al. (2008). They demonstrated that the angle of the first pair of seminal roots varied between Australian wheat varieties. Therefore, we focused on the SRA of the first seminal roots instead of the average angle of the primary, first pair, and second pair of seminal roots for QTL detection.

Previous SRA research has focused mainly on SRA of whole seminal roots or SRA of individual seminal roots (Oyanagi et al. 1993, Manschadi et al. 2008, Hamada et al. 2012, Christopher et al. 2013); however, little is known about relationship between SRAs of individual roots. In this study, we found a significant correlation between SRA of the first pair of roots and SRA of the second pair of roots. However, the correlation coefficient was not high (Fig. 4). This result suggests that the SRA of the first pair of roots and the second pair of roots controlled different genes, at least in part.

Previous studies suggest that SRA could be controlled by multiple genes with small effect (Sanguineti et al. 2007, Christopher et al. 2013, Liu et al. 2013, Richard et al. 2018). In durum wheat, Maccaferri et al. (2016) reported that QTL for SRA was detected at $\mathrm{Xgwm} 169$ at the terminal region of chromosome 6AL. According to their report, QTLs associated with various root traits, including root length, root surface area, and SRA, had formed a cluster in this chromosome region. Alahmad et al. (2019) performed a genome-wide association study using the elite durum wheat populations. They identified a major QTL $(q S R A-6 A)$ that modulates root growth angle and clarified that $q S R A-6 A$ colocated with previously mapped QTLs in durum wheat (Maccaferri et al. 2016). The genomic region that carries $q S R A-6 A$ had many genes related to gravitropism, polar growth, and hormonal signaling
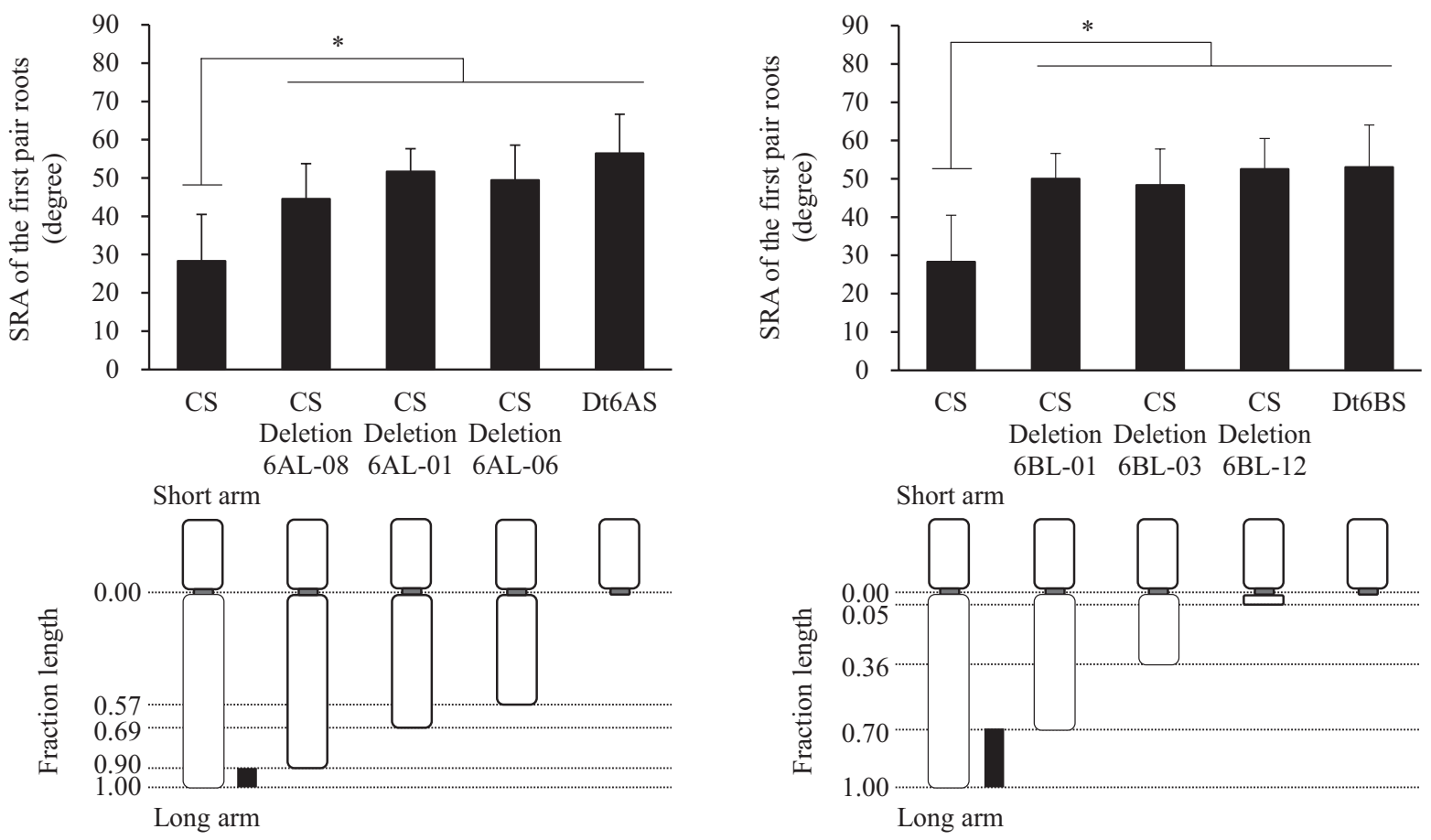

Fig. 5. SRA of CS and CS deletion lines. Open circle with a dotted line shows the break point of long arm that was deficient. The solid bars to the right of open circle indicate QTL regions for SRA of the first pair of seminal roots. * indicates significant difference from CS at the 5\% level (Dunnett's test). 
(Alahmad et al. 2019). In the present study, we found that QSra.obu-6AL is located at the terminal region of chromosome 6AL (FL $=0.9-1.0)$ (Fig. 5). The Xgwm 169 locus, linked to $q S R A-6 A$, was mapped to the region 0.9 to 1.0 of FL (Xie et al. 2012). Thus, there is a possibility that $q S R A-6 A$ and QSra.obu-6AL are at the same locus; however, further study is required to elucidate the relationship between these two QTLs. Furthermore, we detected that QSra.obu-6BL was located at the terminal region of chromosome 6BL. Christopher et al. (2013) identified two QTLs associated with SRA (qRA.qgw-6B and QRA.qgw-6B.2) on chromosome $6 \mathrm{~B}$. The precise chromosomal positions of $q R A$. $q g w-6 B$ and $Q R A . q g w-6 B .2$ are not clear, therefore, it is difficult to clarify whether $Q S$ ra.obu- $6 B L$ and these two QTLs are at the same locus. QSra.obu$6 D L$ may be a novel QTL because no QTL for SRA has been identified on chromosome 6D. Sanguineti et al. (2007) detected QTL for SRA linked to Xgwm234 on chromosome 5BS using a panel of 57 elite durum wheat accessions. Our result revealed that the QSra.obu-5BS is on the short arm of chromosome $5 \mathrm{~B}$. So far, the relationship between these QTLs is unknown.

To develop a crop with a root system architecture adapted to each environment, many researchers have identified QTLs for SRA. However, almost all of QTLs for SRA have not been isolated and no candidate genes were reported. Only DEEPER ROOTING l (DROI) gene, which increases deeper roots in rice, has been isolated by Uga et al. (2013). In a recent research, wheat genome was reported to contain three copies of the DROI orthologs on the long arms of chromosomes 5A (TRIAE_CS42_5AL_TGACv1_374418 A A $119977 \overline{0}), 5 \mathrm{~B}^{-}$(TRIAE_CS $\overline{4} 2 \_5 \mathrm{BL}_{-}^{-}$ TGACv1 405332 AA1325250), and 5D (TRIAE

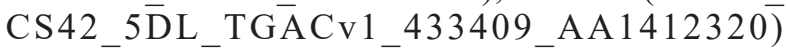
(Kulkarni et al. 2017). It seems that four QTLs (QSra.obu-5BS, QSra.obu-6AL, QSra.obu-6BL, and QSra.obu-6DL) identified in this study are controlled by genes different from the DROI orthologs, because the locus positions on chromosomes are different. Moreover, both QSra. obu-6AL and QSra.obu-6BL located on the terminal regions of the chromosomes $6 \mathrm{AL}$ and $6 \mathrm{BL}$, respectively (Fig. 5). It appears that $Q S r a . o b u-6 A L$ and QSra.obu-6BL may be homoeologous QTLs; however, this remains to be conclusively established. These results suggest that novel genes may be isolated from wheat. The recent release of a wheat reference genome (IWGSC 2018) could be useful in accelerating the gene isolation process for SRA in wheat.

\section{Acknowledgments}

All of materials were provided by the National BioResource Project-WHEAT (NBRP-WHEAT) in Japan. This work was supported by a grant from the Program for Promotion of Basic and Applied Researches for Innovations in Bio-oriented Industry (BRAIN) and JSPS KAKENHI Grant Number $15 \mathrm{~K} 20839$.

\section{References}

Alahmad S, Hassouni KEL, Bassi FM, Dinglasan E, Youssef C, Quarry G, Aksoy A, Mazzucotelli E, Juhasz A, Abel JA, Christopher J, Voss-Fels KP, Hickey LT 2019 A major root architecture QTL responding to water limitation in durum wheat. Front. Plant Sci. 10: 436.

Bassi FM, Sanchez-Garcia M 2017 Adaptation and stability analysis of ICARDA durum wheat elites across 18 countries. Crop Sci. 57: 2419-2430.

Chochois V, Vogel JP, Rebetzke GJ, Watt M 2015 Variation in adult plant phenotypes and partitioning among seed and stem-borne roots across Brachypodium distachyon accessions to exploit in breeding cereals for wellwatered and drought environments. Plant Physiol. 168: 953-967.

Christopher J, Christopher M, Jennings R, Jones S, Fletcher S, Borrell A, Manschadi AM, Jordan D, Mace E, Hammer G 2013 QTL for root angle and number in a population developed from bread wheats (Triticum aestivum) with contrasting adaptation to water-limited environments. Theor. Appl. Genet. 126: 1563-1574.

Endo TR, Gill BS 1996 The deletion stocks of common wheat. J. Hered. 87: 295-307.

Fang Y, Du Y, Wang J, Wu A, Qiao S, Xu B, Zhang S, Siddique KHM, Chen Y 2017 Moderate drought stress affected root growth and grain yield in old, modern and newly released cultivars of winter wheat. Front. Plant Sci. 8: 672.

Hamada A, Nitta M, Nasuda S, Kato K, Fujita M, Matsunaka H, Okumoto Y 2012 Novel QTLs for growth angle of seminal roots in wheat (Triticum aestivum L.). Plant Soil 354: 395-405.

Haque E, Osmani AA, Ahmadi SH, Ban T 2016 Development of pre-breeding technology for root system study and selection of Kihara Afghan wheat landraces (KAWLR) to enhance wheat breeding in the rain-fed region. Breed. Sci. 66: 808-822.

Hassouni KEL, Alahmad S, Belkadi B, Filali-Maltouf A, Hickey L, Bassi F 2018 Root system architecture and its association with yield under different water regimes in durum wheat. Crop Sci. 58: 2331-2346.

IWGSC (The International Wheat Genome Sequencing Consortium) 2018 Shifting the limits in wheat research and breeding using a fully annotated reference genome. Science 361: eaar7191.

Liu X, Li R, Chang X, Jing R 2013 Mapping QTLs for 
seedling root traits in a doubled haploid wheat population under different water regimes. Euphytica 189: 51-66.

Kulkarni M, Soolanayakanahally R, Ogawa S, Uga Y, Selvaraj MG, Kagale S 2017 Drought response in wheat: Key genes and regulatory mechanisms controlling root system architecture and transpiration efficiency. Front. Chem. 5: 106.

Maccaferri M, El-Feki W, Nazemi G, Salvi S, Canè MA, Colalongo MC, Stefanelli S, Tuberosa R 2016 Prioritizing quantitative trait loci for root system architecture in tetraploid wheat. J. Exp. Bot. 67: 11611178.

Manschadi AM, Hammer GL, Christopher JT, Devoil P 2008 Genotypic variation in seedling root architectural traits and implications for drought adaptation in wheat (Triticum aestivum L.). Plant Soil 303: 115-129.

Manschadi AM, Manske GGB, Vlek PLG 2013 Root architecture and resource acquisition - wheat as a model plant. In: Eshel A, Beeckman T, eds. Plant roots-the hidden half, $4^{\text {th }}$ edn. Boca Raton FL: CRC Press, 1-22.

Oyanagi A 1994 Gravitropic response growth angle and vertical distribution of root of wheat (Triticum aestivum L.). Plant Soil 165: 323-326.

Oyanagi A, Nakamoto T, Wada M 1993 Relationship between root growth angle of seedlings and vertical distribution of roots in the fields in wheat cultivars. Jpn.
J. Crop Sci. 62: 565-570.

Passioura JB 2006 Increasing crop productivity when water is scarce-from breeding to field management. Agric. Water Manage. 80: 176-196.

Richard C, Christopher J, Chenu K, Borrell A, Christopher M, Hickey L 2018 Selection in early generations to shift allele frequency for seminal root angle in wheat. Plant Genome 11: 170071.

Sanguineti M, Li S, Maccaferri M, Corneti S, Rotondo F, Chiari,T, Tuberosa R 2007 Genetic dissection of seminal root architecture in elite durum wheat germplasm. Ann. Appl. Biol. 151: 291-305.

Sears ER 1954 The aneuploids of common wheat. Missouri Agric. Exp. Stn. Res. Bull. 572: 1-59.

Uga Y, Sugimoto K, Ogawa S, Rane J, Ishitani M, Hara N, Kitomi Y, Inukai Y, Ono K, Kanno N, Inoue H, Takehisa H, Motoyama R, Nagamura Y, Wu J, Matsumoto T, Takai T, Okuno K, Yano M 2013 Control of root system architecture by DEEP ROOTING 1 increases rice yield under drought conditions. Nat. Genet. 45: 1097-1102.

Xie W, Ben-David R, Zeng B, Dinoor A, Xie C, Sun Q, Roder MS, Fahoum A, Fahima T 2012 Suppressed recombination rate in $6 \mathrm{VS} / 6 \mathrm{AL}$ translocation region carrying the Pm21 locus introgressed from Haynaldia villosa into hexaploidy wheat. Mol. Breed. 29: 399-412. 